Article

\title{
Identification and Comparative Analysis of CBS Domain-Containing Proteins in Soybean (Glycine max) and the Primary Function of GmCBS21 in Enhanced Tolerance to Low Nitrogen Stress
}

\author{
Qingnan Hao ${ }^{1,2}$, Weijuan Shang ${ }^{1,2}$, Chanjuan Zhang ${ }^{1,2}$, Haifeng Chen ${ }^{1,2}$, Limiao Chen ${ }^{1,2}$, \\ Songli Yuan ${ }^{1,2}$, Shuilian Chen ${ }^{2}$, Xiaojuan Zhang ${ }^{2}$ and Xinan Zhou ${ }^{1,2, *}$ \\ 1 Institute of Oil Crops Research, Chinese Academy of Agriculture Sciences, Wuhan 430062, China; \\ haoqingnan@126.com (Q.H.); shang-weijuan2006@163.com (W.S.); zhangchanjuan@aliyun.com (C.Z.); \\ smartchf@163.com (H.C.); ccllmm_008@163.com (L.C.); yyyyy-0909@163.com (S.Y.) \\ 2 Key Laboratory for Biological Sciences of Oil Crops, Chinese Academy of Agricultural Sciences, \\ Ministry of Agriculture, Wuhan 430062, China; chenshuilianjy@163.com (S.C.); xjzhang@oilcrops.cn (X.Z.) \\ * Correspondence: zhouxinan@caas.cn; Tel./Fax: +86-27-8671-1563
}

Academic Editor: Jianhua Zhu

Received: 5 March 2016; Accepted: 20 April 2016; Published: 26 April 2016

\begin{abstract}
Nitrogen is an important macronutrient required for plant growth, and is a limiting factor for crop productivity. Improving the nitrogen use efficiency (NUE) is therefore crucial. At present, the NUE mechanism is unclear and information on the genes associated with NUE in soybeans is lacking. cystathionine beta synthase (CBS) domain-containing proteins (CDCPs) may be implicated in abiotic stress tolerance in plants. We identified and classified a CBS domain-containing protein superfamily in soybean. A candidate gene for NUE, GmCBS21, was identified. GmCBS21 gene characteristics, the temporal expression pattern of the GmCBS21 gene, and the phenotype of GmCBS21 overexpression in transgenic Arabidopsis thaliana under low nitrogen stress were analyzed. The phenotypes suggested that the transgenic Arabidopsis thaliana seedlings performed better under the nitrogen-deficient condition. GmCBS21-overexpressing transgenic plants exhibit higher low nitrogen stress tolerance than WT plants, and this suggests its role in low nitrogen stress tolerance in plants. We conclude that GmCBS21 may serve as an excellent candidate for breeding crops with enhanced NUE and better yield.
\end{abstract}

Keywords: CBS domain; soybean; function analysis; low nitrogen stress tolerance

\section{Introduction}

Nitrogen $(\mathrm{N})$ is one of the main nutrient elements for plant growth and development and thus plays a very important role in plant productivity and crop yield [1]. An appropriate increase in the amount of nitrogenous fertilizer is one of the measures necessary to obtain a high crop yield. However, excessive application of nitrogen fertilizer not only reduces crop nitrogen uptake and utilization efficiency, but also results in reduced crop production efficiency, a waste of resources, environmental pollution and other issues [2,3]. To increase crop yield as a major goal, studies on how to reduce the amount of nitrogen fertilizer and increase crop nitrogen utilization efficiency are very important for the sustainable development of agriculture.

The search to identify genes that improve the nitrogen use efficiency (NUE) of crop plants is ongoing. In our previous study, two soybean genotypes were grown under $\mathrm{N}$-limited conditions; a low $\mathrm{N}$-tolerant variety (Pohuang) and a low $\mathrm{N}$-sensitive variety (84-70) were used for transcriptome analysis. A number of soybean genes were differentially expressed between the two varieties under 
N-limited conditions. Some of these genes may be candidates for improving NUE [4]. One of the genes, GmCBS21, was cloned and analyzed.

The GmCBS21 gene encoded a protein containing the cystathionine beta synthase (CBS) and DUF21 domains [5]. DUF21 domains are transmembrane regions with unknown function adjacent to CBS domains. CBS domains are also widely present in other proteins such as inosine-5'-monophosphate dehydrogenase (IMPDH) [6], voltage-gated chloride channels (CLC) [7] and AMP-activated protein kinase (AMPK) [8]. CBS domains regulate the activity of associated enzymatic and transporter domains in response to binding molecules with adenosyl groups such as AMP and ATP, or $S$-adenosylmethionine [9]. CBS domains are often found in proteins that contain other domains having enzymatic, membrane transporter or DNA-binding activities. However, proteins that contain only CBS domains are also found frequently, particularly in prokaryotes. These standalone CBS domain proteins might form complexes upon binding to other proteins (such as kinases) for interacting with and regulating. The CBS domain-containing proteins (CDCPs) are composed of a superfamily of proteins conserved during evolution. The main feature of these proteins is that they are usually connected to two or four CBS domains [10].

Although the CBS function is unclear, mutations in some human CDCPs lead to genetic diseases [11]. Studies on CDCPs in plants were recently initiated. Some studies revealed that CBSX (protein with only one pair of CBS domains without any other protein domains) proteins directly regulate the activation of thioredoxins and thereby control cellular $\mathrm{H}_{2} \mathrm{O}_{2}$ levels and modulate both plant development and growth [12]. Functional analysis showed that mature CbCBS (CBS domain-containing protein from Coleus blumei) may act as a sensor of cellular energy status and may directly or indirectly regulate cellular energy levels to increase the ATP content in mitochondria during periods of metabolic stress in senescent leaves [13]. Some studies found that many of the CDCPs with unknown function in Arabidopsis and Oryza were related to abiotic stress [10]. Singh et al. found that OsCBSX4 was regulated by stress and development, and overexpression of OsCBSX4 in model tobacco (Nicotiana tabacum) plants resulted in enhanced tolerance against various abiotic stresses such as salinity, heavy metals, and oxidative stress [14]. OsBi1 is a CBS-containing gene that is implicated in the resistance of rice plants to brown plant hoppers and responds to biotic and abiotic stress [15].

In this study, we identified and carried out a comparative analysis of CBS domain-containing proteins in soybean. Furthermore, one of the genes, GmCBS21, was cloned and its implication in low nitrogen stress tolerance was analyzed. GmCBS21 overexpression in Arabidopsis thaliana plants induced higher tolerance to low nitrogen conditions, suggesting a role in nitrogen stress responses.

\section{Results}

\subsection{Identification, Characterization and Classification of CBS Domain-Containing Proteins in Soybean}

Based on the domain sequences of the CBS superfamily members of Arabidopsis and rice, we searched the CBS genes in the soybean database of NCBI by BLASTP, and searched in the phytozome soybean genome database using the keyword (CBS domain). A total of 71 putative CBS genes were identified by removing redundant sequences and different transcripts of the same gene. All these putative CBS protein sequences were predicted to include one or two CBS domains. The 71 soybean CBS genes were named GmCBS1 to GmCBS71 according to their chromosomal positions. Detailed information about soybean CBS genes is given in Table S1. The gene-encoded peptides' length ranged from 166 (Glyma.16G086600) to 801 (Glyma.11G004600) amino acids, Mw from 18.6 to $88.2 \mathrm{kDa}$ and pI from 4.90 to 9.30. Subcellular localization prediction indicated that 24 genes were localized in the plasma membrane, 21 genes were localized in the chloroplast, 19 genes were localized in the cytoplasmic, four genes were localized in the nuclear, two genes was localized in the mitochondrial, and one gene was localized in the endoplasmic reticulum.

In previous studies of Arabidopsis and rice, all the CDCPs were divided into two groups: one CBS domain-containing proteins and two CBS domain-containing proteins. One CBS domain-containing 
proteins were further classified into seven subgroups based on the additional domain(s) present in their sequences [10]. Of the 71 soybean CBS identified genes, 10 presented a single CBS domain whereas 15 contained two CBS domains. All the other members of the group presented additional domains different to the CBS domain. Thus, three genes contained a domain of unknown function (DUF21) (PF01595) and a CorC_HlyC domain (PF03471) at the N-terminus and C-terminus of the proteins, respectively, whereas 12 members contained the voltage-gated chloride channel domain (or motif) at the N-terminus and two genes the pentatricopeptide repeat (PPR) motif. Finally seven genes, two genes and one gene presented Phox/Bemp1 (PB1), inosine-5'-monophosphate dehydrogenase (IMPDH) (PF00478) or the sugar isomerase (SIS) domains, respectively.

\subsection{Phylogenetic Motif Recognition, Genome Distribution and Gene Structure of the CBS Superfamily Genes in Soybean}

To determine the phylogenetic relationships among the different members of the CBS superfamily in soybean, phylogenetic analysis based on alignments of the 71 full-length CBS protein sequences was performed. The phylogenetic analysis indicated that 71 CBS superfamily members can be divided into nine groups (Figure 1A). The structure of the corresponding genes was shown in Figure 1B.
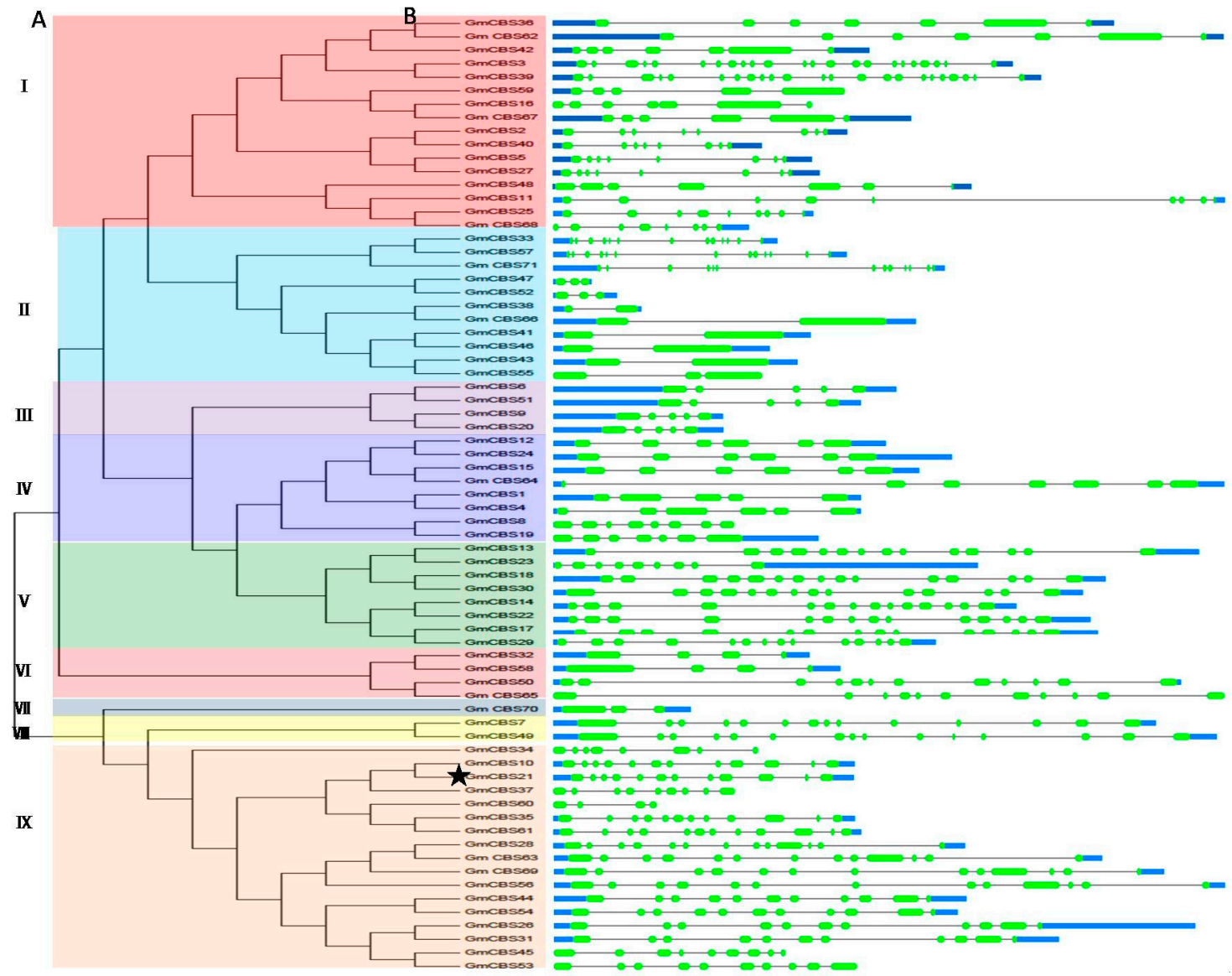

Figure 1. Phylogenetic analysis and structure of soybean CBS genes. (A) Phylogenetic tree construction of soybean CBS genes based on the full-length deduced amino acid sequences using MEGA 5.0 (Koichiro Tamura, Daniel Peterson, Nicholas Peterson, Glen Stecher, Masatoshi Nei, and Sudhir Kumar) by the maximum likelihood method with 500 bootstrap replicates. The tree showed nine major phylogenetic groups (group I to IX) indicated by different colored background. The star marked our candidate gene GmCBS21; (B) Exon/intron structures of GmCBS genes. Green boxes represent exons, black lines indicate introns, and blue boxes represent untranslated region (UTR). 
The members within each group showed similar exon/intron structures. Group I comprised 16 members, and most of them are voltage-gated chloride channel proteins except GmCBS2, GmCBS40, GmCBS5 and GmCBS27. Group II was formed by 11 members which are all AMP-activated protein kinases. Group III was formed by four members with five exons. Group IV was constituted by eight members, which may be AMP-activated protein kinase. Group V was formed by eight members with 14 exons, except GmCBS23, which has nine exons. Group VI was formed by four members. Group VII was constituted by one member with three exons, which contain a SIS domain. Group VIII was formed by two members with 14 and 15 exons. Group IX was formed by 17 members which are all proteins of unknown function. Several pairs of GmCBS proteins have a high degree of homology in the terminal nodes, and they are putative paralogous pairs. A total of 30 putative paralogous pairs were identified, with sequence identity ranging from $65 \%$ to $99 \%$ (Table S2).

To further reveal the diversification of CBS genes in soybean, putative conserved motifs were predicted by the program MEME. A total of 20 distinct motifs were identified in all 71 CBS domain-containing proteins. The schematic distribution of the 20 motifs among the different gene groups is shown in Figure 2, and the identified multilevel consensus sequence for the motifs is shown in Table S3. Motif 1, motif 2 and motif 8 mainly existed in group VI and group IX, and were TlyC CBS_pair_CorC_HlyC. Motif 3 and motif 4 mainly existed in group VI and group IX, and had the DUF domain. Motif 5 was present in most of the GmCBS domains (except GmCBS70 and GmCBS37), and was CBS_pair_29. Motif 6 was present in most of the GmCBS domains (except GmCBS35, GmCBS45, GmCBS60 and GmCBS61), and was CBS_pair_10. Motif 7, motif 10, motif 13, motif 14 and motif 16 were present in group I, and were Voltage_gated_ClC. Motif 9 was CBS_pair_CAP-ED_DUF294_PBI_assoc, and was present in the group I, group V and group VI. Motif 15 was present in group V, and was CBS_pair_CAP-ED_DUF294_PBI_assoc. Motif 18 was present in group V, and was PB1. Motif 18 was present in group II, group IV and group V, and was CBS_pair_10.

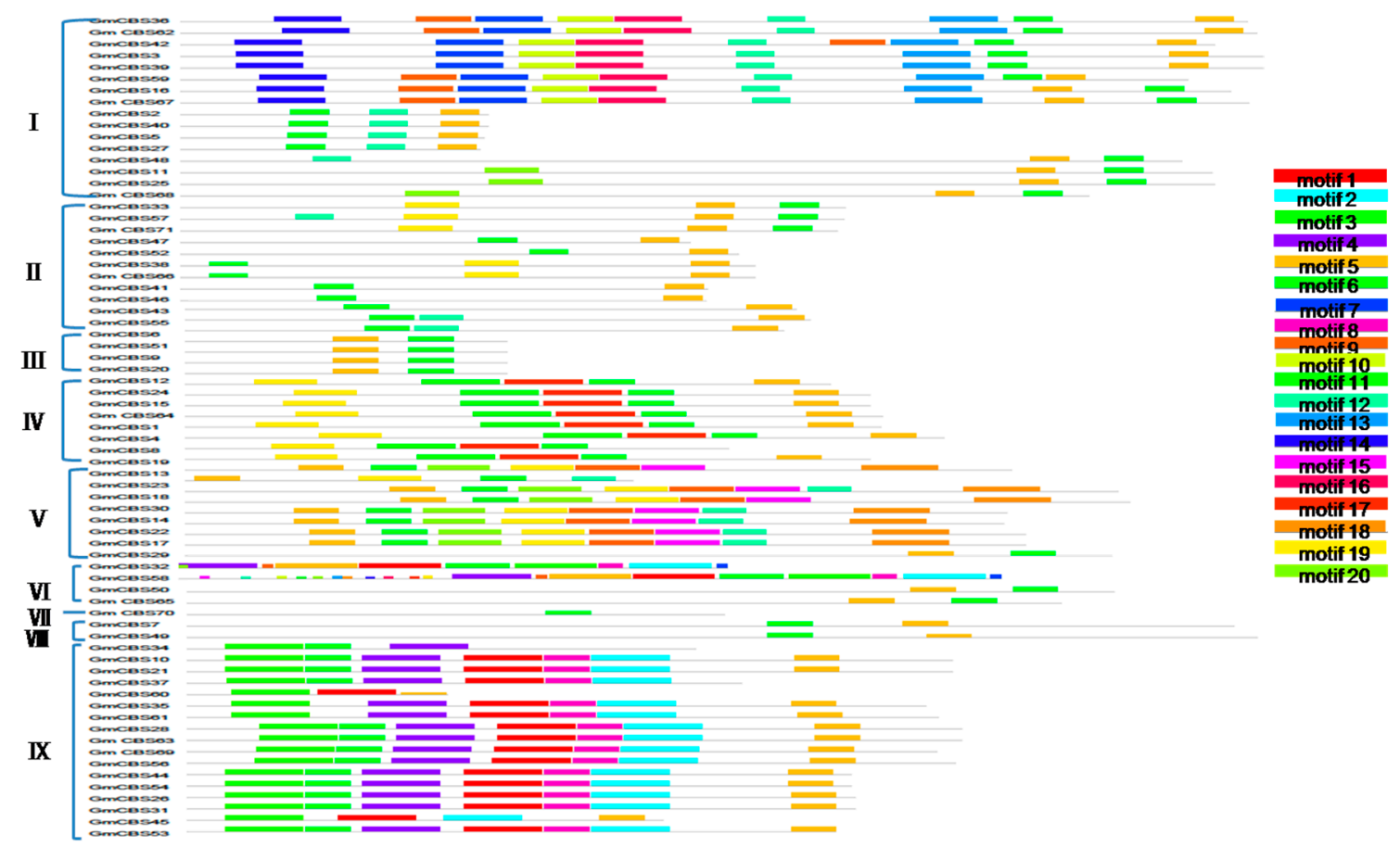

Figure 2. Schematic distribution of the conserved motifs in soybean CBS superfamily by MEME. The conserved motifs were identified in the proteins of every group. Each colored box below the tree represents the conserved motifs. Motif 2, 5, 6, 8, 9, 15 and 19 were CBS-related motifs.

The physical locations of the CBS genes on soybean chromosomes are shown in Figure 3. Seventy-one soybean CBS genes were unevenly distributed on 18 chromosomes, except for 
chromosomes 3 and 10. Among these chromosomes, chromosome 6 and chromosome 15 had the largest number of CBS genes with seven, followed by chromosomes 4, 9, 13 with six. However, chromosomes $12,18,20$ only had one CBS gene.
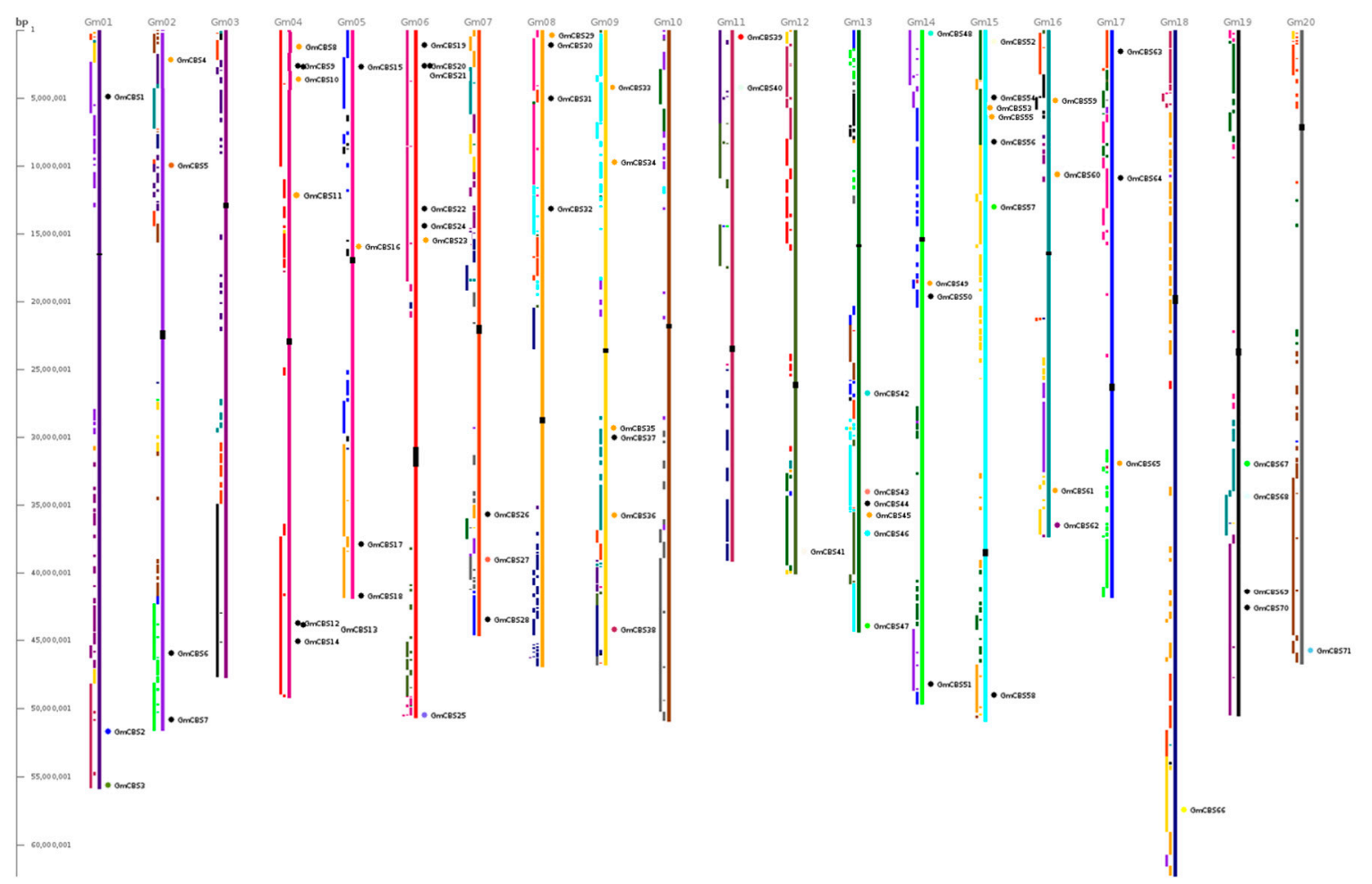

Figure 3. Chromosomal location and region duplication of GmCBS genes. The schematic diagram of genome-wide chromosome organization and segmental duplication was made from the CViT genome search and synteny viewer at the Legume Information System. Colored blocks to the left of each chromosome show duplications with chromosomes of the same color.

\subsection{The Expression Pattern and Subcellular Localization of GmCBS21}

In our former Digital Gene Expression (DGE) data libraries (N-tolerant variety (Pohuang) vs. low $\mathrm{N}$-sensitive variety (84-70)), GmCBS21 was a differentially expressed gene under $\mathrm{N}$-limited conditions (Table S4). qRT-PCR analysis reconfirmed the low nitrogen stress-induced upregulation in soybean, with a higher relative expression in Pohuang compared with 84-70 (Figure 4). The function of a protein is tightly correlated with its expression pattern and subcellular localization. First, using qRT-PCR, we investigated the expression level of GmCBS21 in different organs of Pohuang. qRT-PCR showed that the GmCBS21 gene was expressed in the leaves, stems, and roots (Figure 5A). The GmCBS21 gene was significantly upregulated after low $\mathrm{N}$ treatment in leaves and roots, but was downregulated in stems. The expression of GmCBS21 was significantly upregulated in leaves and roots at $6 \mathrm{~h}$ and 12 days. In stems, the expression of GmCBS21 was significantly downregulated at $0.5,9 \mathrm{~h}$, and 12 days (Figure 5A). These results indicate that GmCBS21 may be involved in the response to low $\mathrm{N}$ stress. 

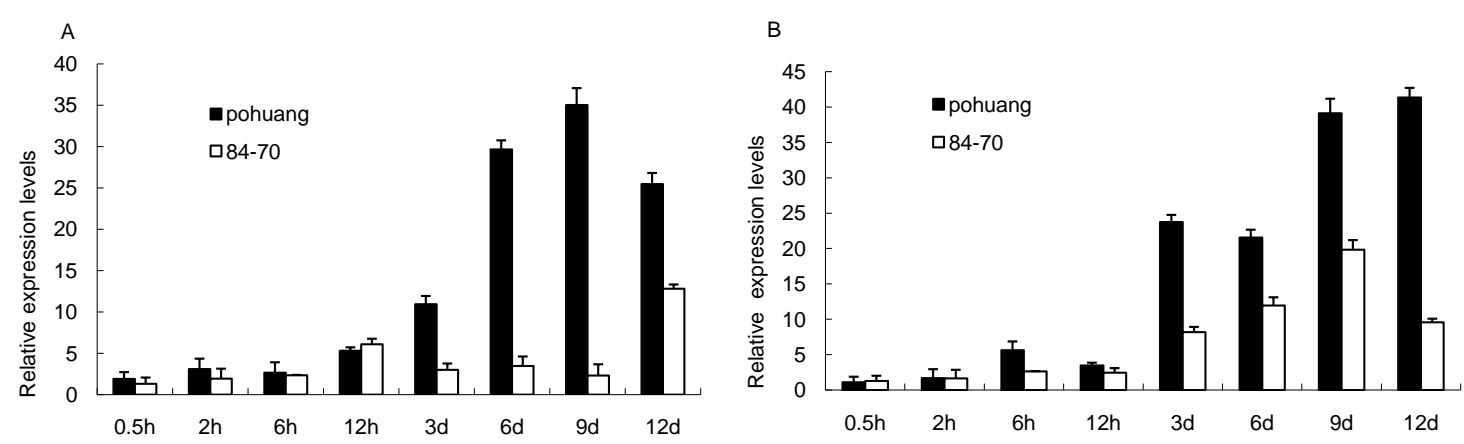

Figure 4. The GmCBS21 transcript is induced by low nitrogen stress: (A) The expression in leaf and stem; (B) The expression in root. Three independent experiments were performed, and values are shown as means and error bars indicate standard deviation $(n=3)$.

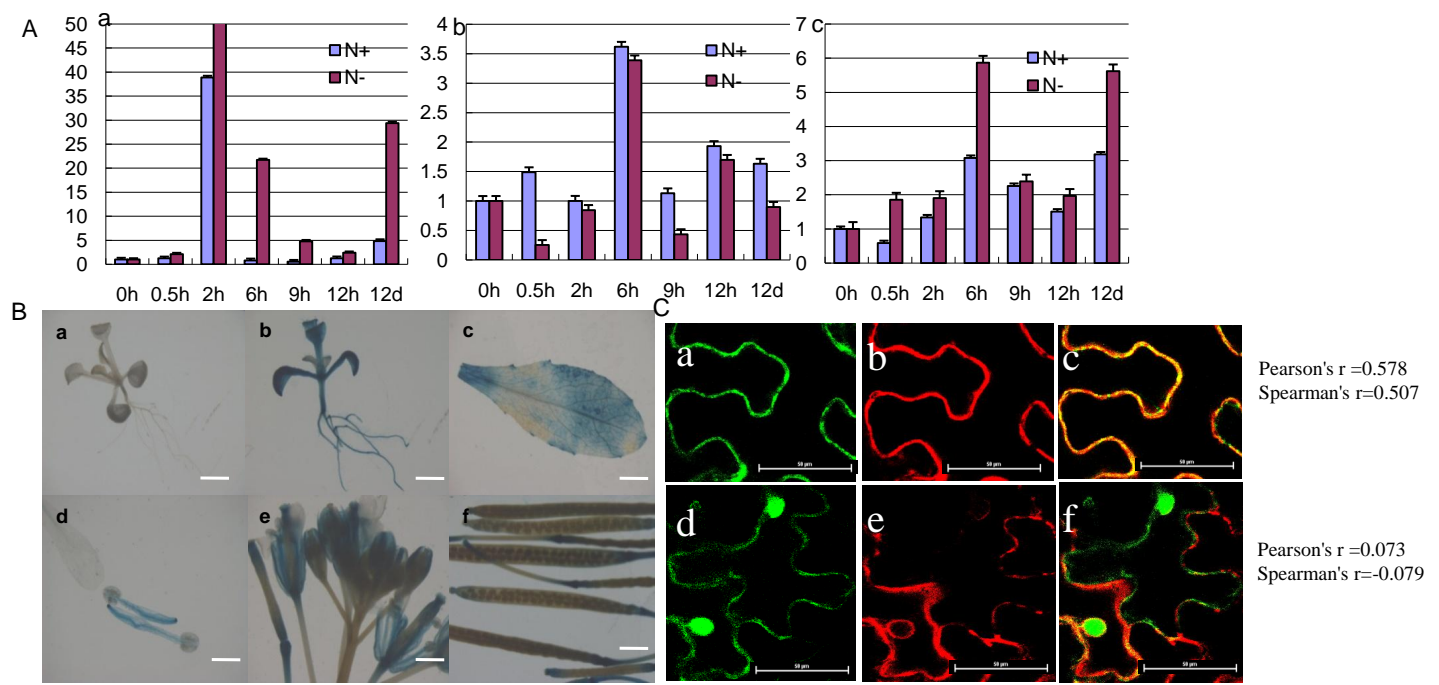

Figure 5. Expression patterns of GmCBS21 and subcellular localization of GmCBS21. (A) Relative expression profiles of soybean GmCBS21 in various organs. Soybean seedlings (Pohuang) were treated with normal nitrogen $(\mathrm{N}+)$ and low nitrogen $(\mathrm{N}-$ ); samples were taken at different time (after treatment $0,0.5,2,6,9,12 \mathrm{~h}, 12$ days). (a) Relative expression profiles of soybean GmCBS21 in leaves; (b) Relative expression profiles of soybean GmCBS21 in stems; (c) Relative expression profiles of soybean GmCBS21 in roots. N+ was basic Hoagland's nutrient solution. N- was basic Hoagland's nutrient solution with $1 / 10 \mathrm{~N}$ concentration. Three independent experiments were performed, and values are shown as means and error bars indicate standard deviation $(n=3)$; (B) Expression of the GmCBS21:GUS reporter gene in transgenic Arabidopsis plants. (a) Expression of the GmCBS21:GUS reporter gene in seedlings of wild type; (b) Expression of the GmCBS21:GUS reporter gene in transgenic seedlings; (c) Expression of the GmCBS21:GUS reporter gene in transgenic leaf; (d) Expression of the GmCBS21:GUS reporter gene in transgenic stamens; (e) Expression of the GmCBS21:GUS reporter gene in transgenic flower; (f) Expression of the GmCBS21:GUS reporter gene in transgenic siliques; Scale bars: $1 \mathrm{~cm}(\mathbf{a}-\mathbf{c})$; $1.5 \mathrm{~cm}(\mathbf{d}, \mathbf{e}) ; 0.5 \mathrm{~cm}(\mathbf{f})$; (C) Subcellular localization of the GmCBS21 protein. Subcellular localization of the fused pJG053-35s::GmCBS21 -GFP in tobacco leaves cells. The pJG053::GFP construct was used as the control. (a) Green Fluorescent Protein (GFP )channel showing GmCBS21::GFP expression; (b) Red Fluorescent Protein (RFP) channel showing pm-rk CD3 mCherry marker expression; (c) co-localization of GmCBS21::GFP with the pm-rk CD3-1007 mCherry marker; (d) GFP channel showing pJG053::GFP expression; (e) RFP channel showing pm-rk CD3 mCherry marker expression; (f) co-localization of pJG053::GFP with the pm-rk CD3-1007 mCherry marker. Statistical analysis of the co-localization was done using Pearson's (rP) and Spearman's (rS) correlation factors [16]. Bar $=50 \mu \mathrm{m}$. 
In order to examine the tissue expression patterns of GmCBS21, we performed histochemical expression analysis of the gene using transgenic Arabidopsis plants carrying promoter-GUS fusion report systems. T3 homozygous transgenic plants were used for the GUS assays. The results showed that GUS activity was detected in seedlings and all tissues and organs examined, including the leaf, root, stamens, flower, and siliques (Figure 5B).

To determine the cellular localization of the GmCBS21 protein, we introduced the pJG053-35s: GmCBS21-GFP and pJG053-GFP constructs into tobacco leaves cells using Agrobacterium-mediated transformation. Green fluorescence of pJG053-35s:GmCBS21-GFP occurred mainly in the plasma membrane, whereas that of pJG053-GFP alone was distributed throughout the cell (Figure 5C).

\subsection{Prokaryotic Expression and Cystathionine $\beta-S y n t h a s e$ Activity Assay}

In order to measure the cystathionine $\beta$-synthase of GmCBS21 in vitro, we expressed and purified the protein using a bacterial expression system. The protein was expressed in soluble form with a higher protein activity. The purified proteins were analyzed using SDS-PAGE (Figure S1).

The purified proteins were used to determine cystathionine $\beta$-synthase activity. The results are shown in Table S5. The assay group absorbance value and the control group absorbance value were similar. The specific activity of purified cystathionine $\beta$-synthase was $0.37 \mathrm{U} / \mathrm{mg}$ (specific activity). Based on this value, it can be considered that the protein does not have cystathionine $\beta$-synthase activity.

\subsection{Phenotype of GmCBS21 Overexpression Transgenic Seedlings under Low Nitrogen Stress}

A GmCBS21 overexpression vector was constructed (Figure S2A) and used to transform Arabidopsis, resulting in GmCBS21-OXP seedlings. RT-PCR showed that GmCBS21 was constitutively expressed in all 11 T2 Arabidopsis lines (Figure S2B). These findings indicated that all 11 lines would be useful in the analysis of stress tolerance. L1, L3, and L8 were chosen for further analysis.

Low nitrogen tolerance was evaluated in both seedlings and adult plants. In nitrogen-deficient medium, non-transgenic (NT) and transgenic lines (TL) seedlings exhibited the typical nitrogen-deficient phenotype consisting in discoloration of leaves and less vigorous growth. However, this deficient phenotype was clearly much reduced in TL plants (Figure 6A). In adult plants, all three transgenic lines had significantly larger rosettes than the wild type after nine days of treatment in low nitrogen solution (Figure 6B). The phenotypes suggested that TL performed better under the nitrogen-deficient condition.

\subsection{Physiological Indexes of GmCBS21 Overexpression Transgenic Seedlings under Low Nitrogen Stress}

The fresh weight, total nitrogen, ammonium nitrogen, nitrate nitrogen, and non-protein nitrogen concentrations were measured in NT and TL plants. First, we compared the physiological indexes of soil-grown NT and GmCBS21 overexpression plants under a normal nitrogen condition (HN). Results showed that the fresh weight of TL was higher than NT, with a maximum 1.23-fold increase in L3 relative to the NT (Figure 6C, panel a). The results of total nitrogen, ammonium nitrogen, nitrate nitrogen, and non-protein nitrogen concentrations are shown in Figure 6C, panels b-e N+. There was no significant difference between the NT and TL plants. After nine days of treatment in low nitrogen solution, the fresh weight was lower than that measured, and TL had a higher weight than the NT, with a maximum 1.43-fold increase in L1. The concentrations of all types of nitrogen were higher in TL than NT. Specifically, the total nitrogen and nitrate nitrogen concentrations were significantly higher, i.e., 1.31- to 1.70-fold and 1.32- to 1.74-fold higher, respectively, in the transgenic lines overexpressing GmCBS21 compared with the control (Figure 6C, panels b-e N-).

During plant growth, sugar and nitrogen metabolism may regulate and control each other. In this study, more soluble sugar accumulated in NT and TL in LN treatment than in HN treatment, and the soluble sugar content of TL plants was slightly lower than that of the NT plants in the LN treatment 
(Figure 6C, panel f). No obvious differences were observed in the soluble sugar concentrations of NT and TL plants in HN treatment (Figure 6C, panel f).

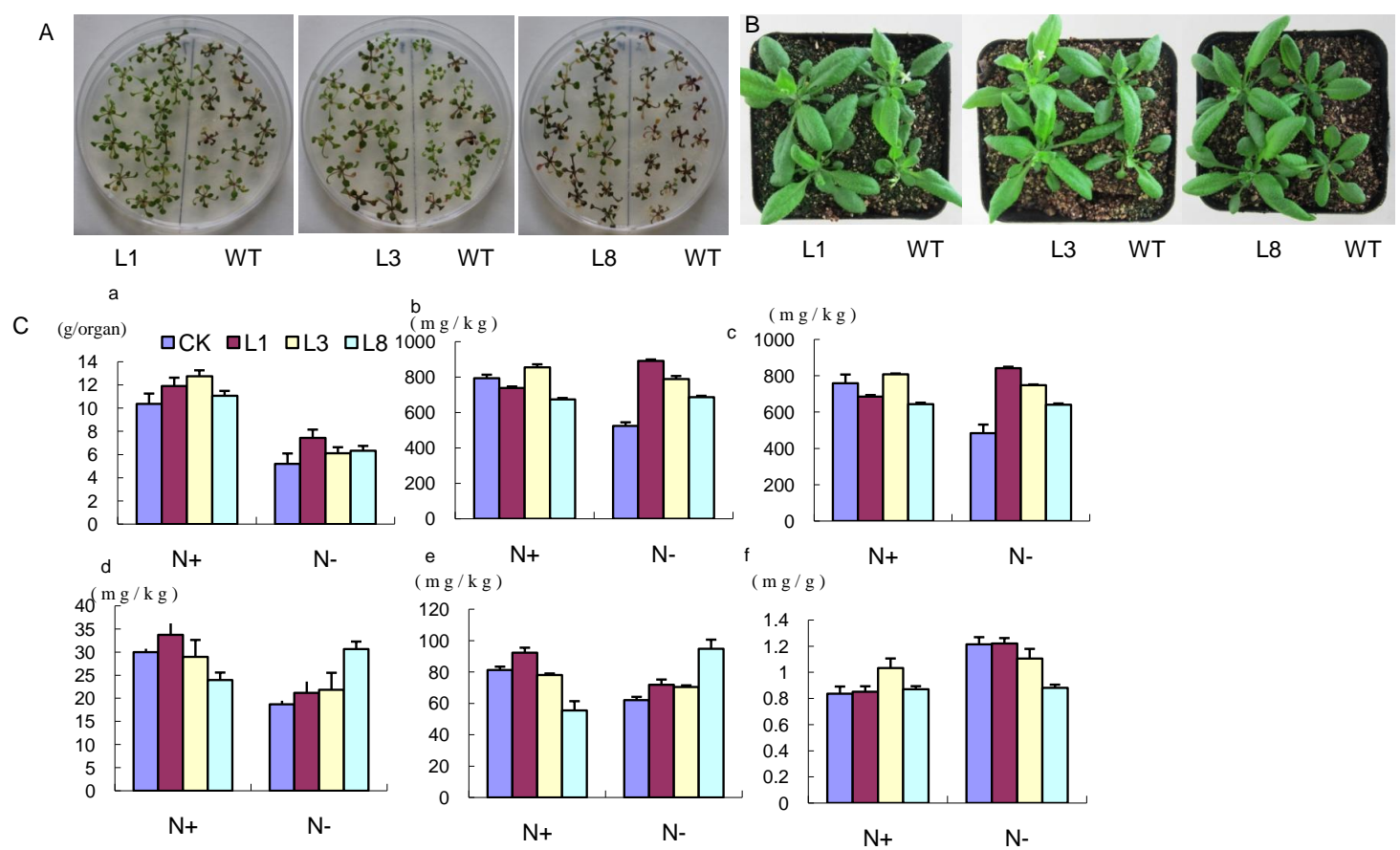

Figure 6. Enhanced low nitrogen stress tolerance in plants overexpressing GmCBS21. (A) The phenotype of transgenic plants and wild-type plants grown in low nitrogen medium. L1, L3 and L8 were transgenic lines. WT was wild-type control line; (B) The phenotype of transgenic plants and wild-type plants grown in low nitrogen vermiculite; (C) Several physiological indexes of transgenic plants and wild-type plants grown in low $\mathrm{N}$ and normal $\mathrm{N}$ condition. L1, L3 and L8 were transgenic lines. CK was wild-type control line. (a) Fresh weight of wild-type and GmCBS21 plants; (b) Total nitrogen concentration of wild-type and GmCBS21 plants; (c) Nitrate-N concentration of wild-type and GmCBS21 plants; (d) Ammonium nitrogen concentration of wild-type and GmCBS21 plants; (e) Non-protein nitrogen concentration of wild-type and GmCBS21 plants; (f) Soluble sugar contents of wild-type and GmCBS21 plants. L1, L3 and L8 were transgenic lines. WT was wild-type control line. Arabidopsis thaliana seedlings were treated with normal nitrogen $(\mathrm{N}+)$ and low nitrogen $(\mathrm{N}-)$. Seven-day-old plants grown on 1/2 MS medium were transferred to vermiculite supplied with $1 / 2$ Hoagland's solution and left to bolt (two weeks). Then, they were transferred to normal $1 / 2$ Hoagland's solution and low nitrogen 1/2 Hoagland's solution, respectively. After nine days in the low nitrogen solution, all plants were harvested. Data represent as the mean value \pm S.E. of three replicates.

\subsection{Effects of GmCBS21 Expression on Amino Acid Composition}

To evaluate the effects of GmCBS21 expression on nitrogen assimilation, the free amino acids in NT and TL plants were analyzed. The total free amino acid concentration of TL plants was higher than that of NT (Figure 7A). The concentrations of Asp, Glu, Gly, Ala, Met, Leu, Phe, Lys, His, Pro, and Asn were higher in TL than NT, and the concentrations of Ser, Cys, Tyr and Gln were lower in TL than NT under HN treatment (Figure 7B). Under LN stress, the concentrations of Asp, Thr, Glu, Gly, Ala, Met, Tyr, Lys, His, Pro, Gln, and Asn were higher in TL than NT, and the concentrations of Ser, Cys, and Ile were lower in TL than NT (Figure 7C). Glutamine and glutamate are good markers for nitrogen utilization. Increases in both Asn and Gln concentrations in the phloem sap during senescence can cause amino acid accumulation, and these residues are known to play a key role in rendering $\mathrm{N}$ available for remobilization from senescing leaves. Although increased amounts of several other amino acids were observed, the increases in Glu, Gln, and Asn were remarkable. 

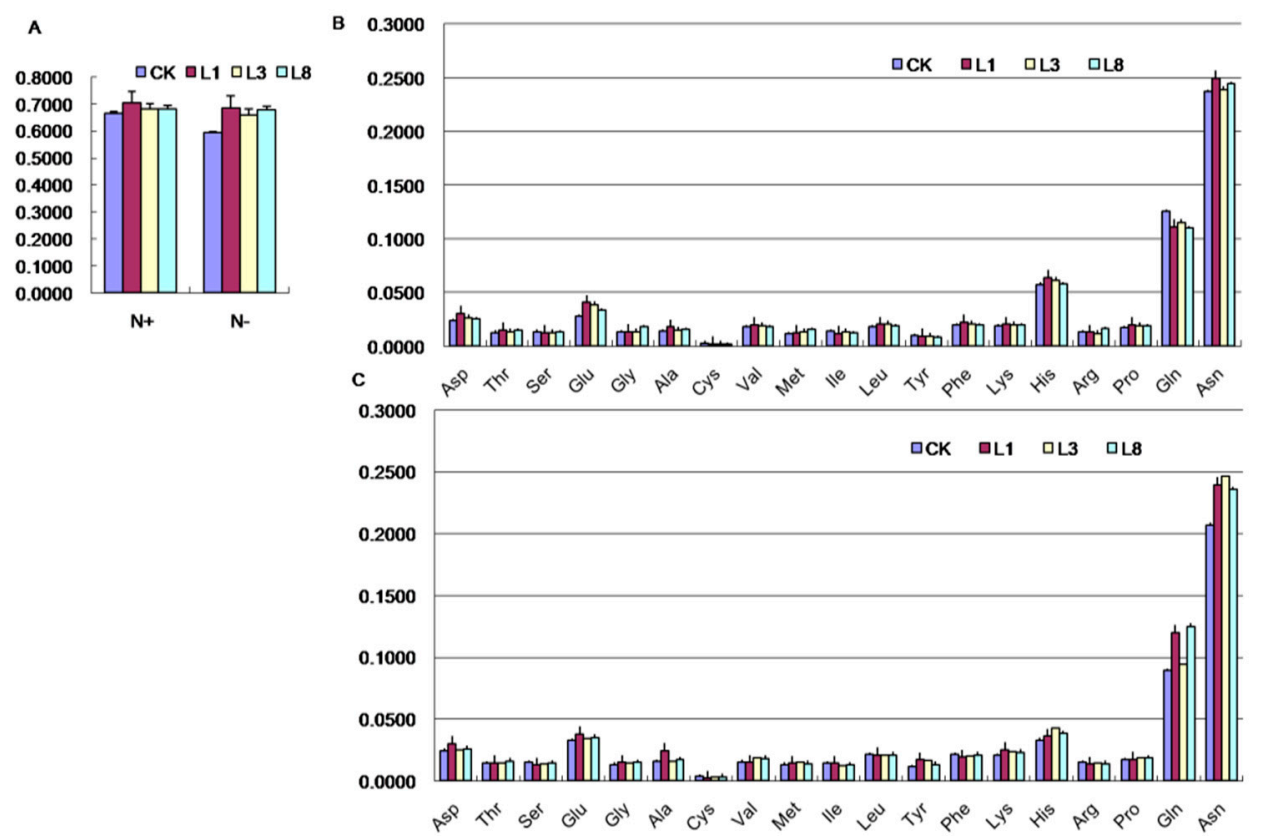

Figure 7. Concentrations of amino acids in transgenic and wild-type plants: (A) Concentrations of total amino acids; (B) Amino acid analysis at the normal N level; (C) Amino acid analysis at the low N level. L1, L3 and L8 were transgenic lines. CK was wild-type control line. Arabidopsis thaliana seedlings were treated with normal nitrogen $(\mathrm{N}+)$ and low nitrogen $(\mathrm{N}-)$. Data represent the mean value \pm S.E. of three replicates.

\section{Discussion}

The cystathionine-synthase (CBS) domain-containing proteins (CDCPs) consist of a large superfamily of evolutionarily conserved proteins, and these are present in all life science fields. Most of the CDCPs have been studied thus far in humans or other animals [11]. In plants, CDCPs have been reported infrequently, and hence their occurrence and possible function still need to be explored.

In recent years, some CDCPs such as IMPDH [15,17], AMPK [18] and ClC [19-21] have been studied in plants. However, GmCBS21 has not been studied. At present, CDCPs with known functions that have been studied in soybean include IMPDH [22], $\mathrm{ClC}$ [23,24], and AMP-activated protein kinase. In order to determine the relationship between GmCBS21 and other CDCPs, a phylogenetic tree of the soybean CDCPs was constructed (Figure 2A). Our candidate protein, which is named GmCBS21, has low homology to other known proteins. The protein belongs to an independent unknown function branch. Therefore, exploring the function of GmCBS21 is meaningful.

In this paper, to validate one of the CDCPs of unknown function, GmCBS21, a gene encoding a protein with a cystathionine-synthase (CBS) domain, was cloned from both 84-70 and Pohuang soybean because this gene was found to be differentially regulated (higher expression in Pohuang) among the contrasting genotypes in our previous study [4]. However, based on the sequencing result, no difference was found in the CDS or peptide sequence for the GmCBS21 alleles between 84-70 and Pohuang (data not shown). These results indicate that higher expression levels of this gene in Pohuang might play a role in its low nitrogen tolerance.

CDCPs may play an important role in the development and stress response/tolerance of plants with respect to salinity, heavy metals, and oxidative stress [10]. However, the role of CDCPs in low nitrogen stress has not been investigated. In this study, the first description of an improvement in NUE under a nitrogen-deficient condition is the overexpression of GmCBS21 in Arabidopsis. Three independent T2 homozygous lines were selected for further molecular and physiological analysis. When growing Arabidopsis plants for nine days under low nitrogen conditions, the transgenic lines with elevated GmCBS21 expression had an improved phenotype compared with the control 
plants (Figure 6A). This included an increase in the fresh weight as well as the nitrogen concentration (Figure 6C).

Based on this correlative evidence between transcript accumulation and low nitrogen tolerance, we proposed that GmCBS21 might play an important role in low nitrogen tolerance in plants. However, the mechanisms of low nitrogen tolerance conferred by GmCBS21 overexpression plants remain unclear. We investigated the expression of several known genes involved in several main processes of nitrogen utilization in transgenic plants. The full name and the TAIR (The Arabidopsis Information Resource) coding number of these genes are shown in Table S6. The results are shown in Figure 8. The main frame of Figure 8 was based on previous work [25].

The main $\mathrm{N}$ forms absorbed by plants are water-soluble nitrate $\left(\mathrm{NO}_{3}{ }^{-}\right)$, ammonium $\left(\mathrm{NH}_{4}{ }^{+}\right)$, and to a lesser extent, proteins, peptides or amino acids [2]. The $\mathrm{N}$ uptake process requires the involvement of transporters. Dual-affinity nitrate transporter NRT1.1 is a transceptor that senses the external nitrate concentration and activates ANR1 (a MADS-box gene), which mediates the nitrate-signaling pathway to regulate nitrate-stimulated lateral root development [26,27]. The expression levels of NRT1.1 and ANR1 were increased in the GmCBS21 transgenic plants compared with the wild type under conditions of normal (sufficient $\mathrm{N}$ ) and low nitrogen (deficient N) (Figure 8). Cytokinins regulate NRT expression and act as an $\mathrm{N}$ satiety signal to decrease nitrate uptake by roots [28]. Nitrate-inducible IPT3 (adenosine phosphate iso-pentenyl-transferase 3) is a key determinant of nitrate-dependent cytokinin biosynthesis [29]. The expression levels of CKX2 and IPT3 were increased in the GmCBS21 transgenic plants compared with the wild type under conditions of normal and low nitrogen. Ammonium uptake is facilitated by AMT transporters. The expression levels of AMT1 were lower in the GmCBS21 transgenic plants compared with the wild type under two nitrogen conditions (Figure 8). Plant roots can absorb small amounts of amino acids from the soil. Amino acid transporters play a role in this process. The expression levels of AAT1 were increased in the GmCBS21 transgenic plants compared with the wild type under conditions of low nitrogen, and were reduced under conditions of sufficient $\mathrm{N}$.
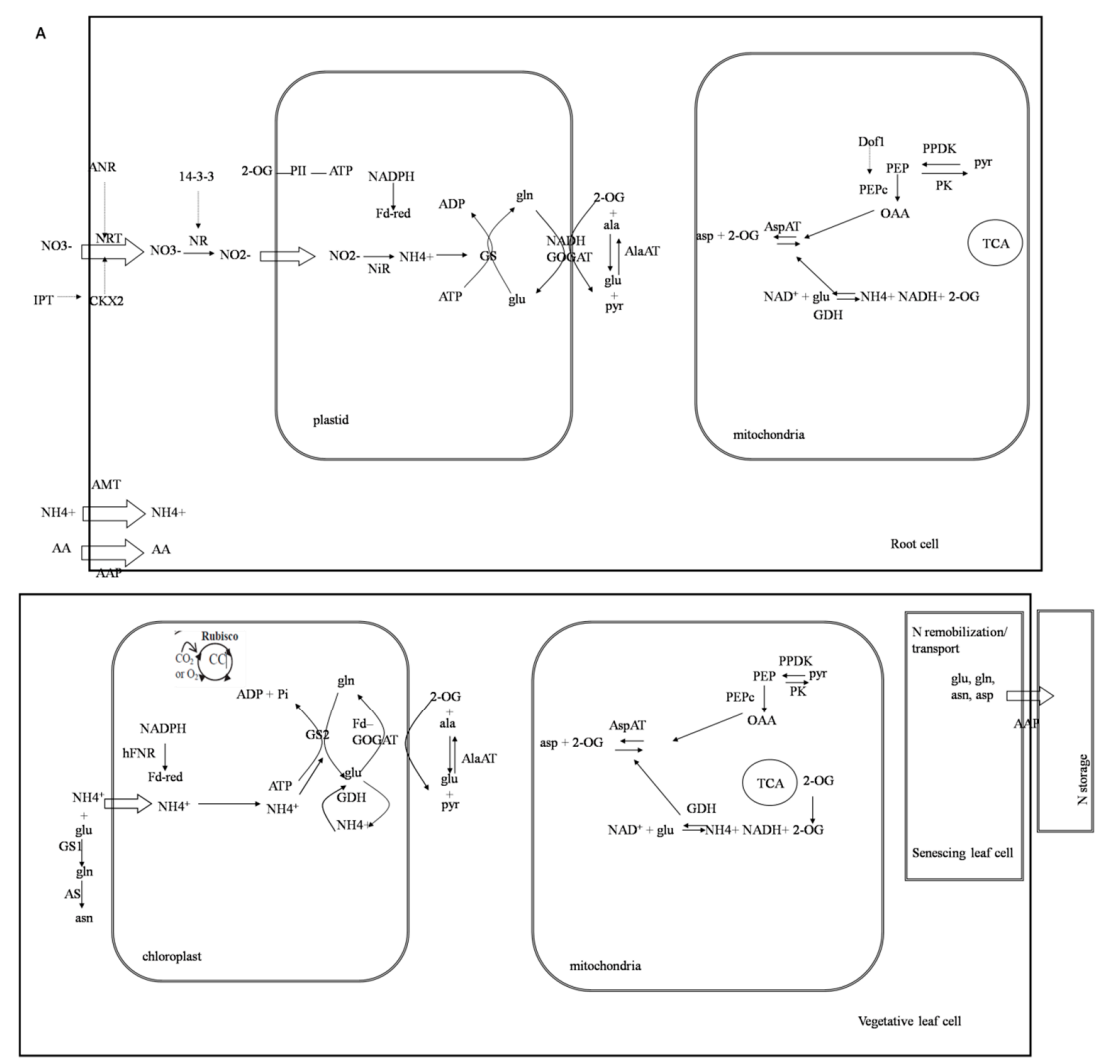

Figure 8. Cont. 

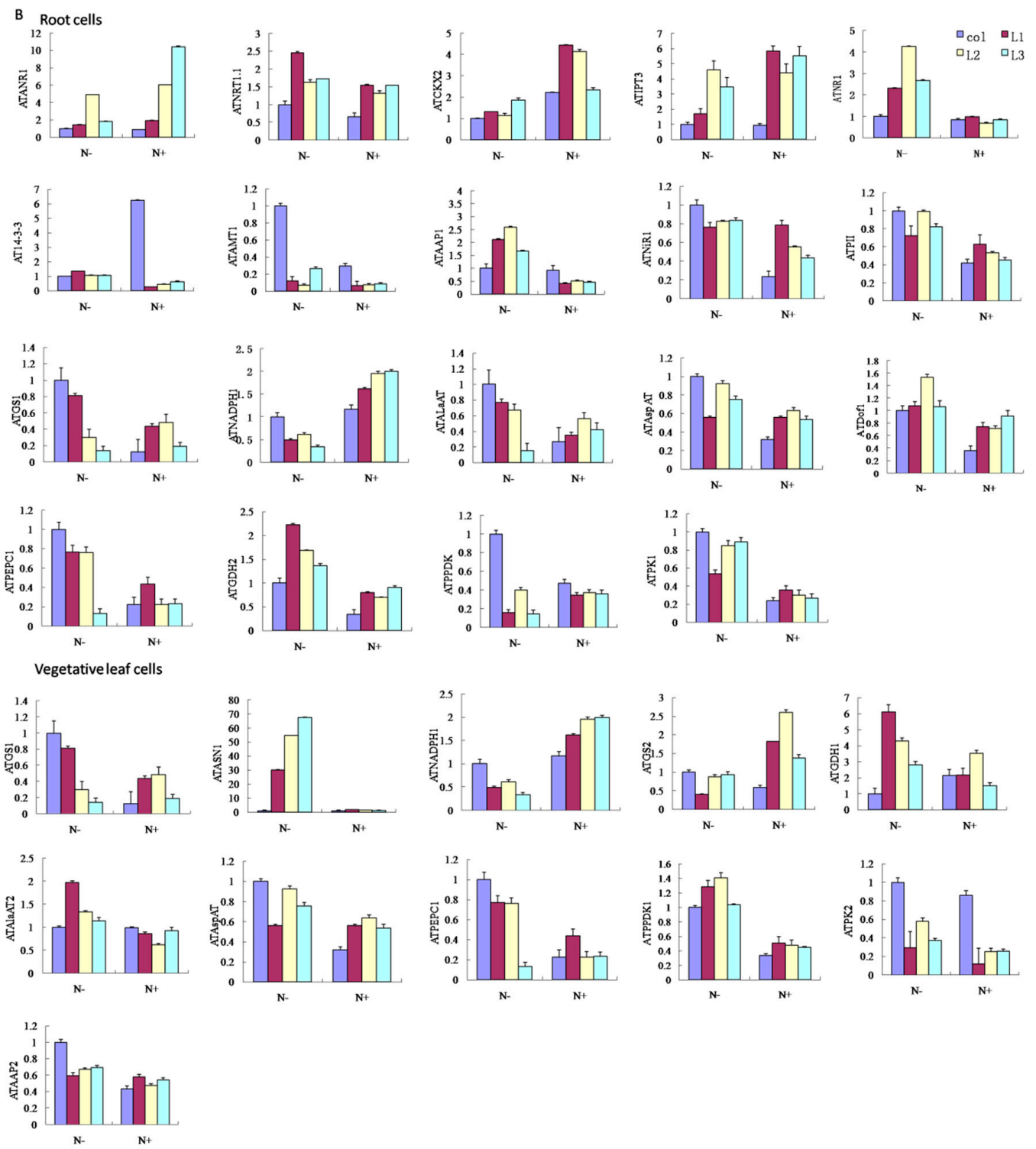

Figure 8. (A) The main processes of nitrogen utilization in plants; Dashed arrows represent transcript regulation, hollow arrows represent transport across membranes and black solid arrows represent an enzymatic reaction (B) The expression of genes involved in nitrogen uptake, assimilation, and remobilization of transgenic plants. L1, L3 and L8 were transgenic lines. Col was wild-type control line. AA, amino acids; AAT, amino acid transporter; AMT, ammonium transporter; NRT, nitrate transporter; 2-OG, 2-oxoglutarate; PK, pyruvate kinase; GS, glutamine synthetase; NR, nitrate reductase; AspAT, aspartate aminotransferase; $\mathrm{PEPc}$, phosphoenolpyruvate carboxylase; $\mathrm{GDH}$, glutamate dehydrogenase; AlaAT, alanine aminotransferase; IPT, isopentenyl transferase; CKX, cytokinin oxidase/dehydrogenase. L1, L3 and L8 were transgenic lines. Col was wild-type control line. Arabidopsis thaliana seedlings were treated with normal nitrogen $(\mathrm{N}+)$ and low nitrogen $(\mathrm{N}-)$. Data represent the mean value \pm S.E. of three replicates.

Nitrogen assimilation occurs after nitrogen has been taken up by higher plants. $\mathrm{NO}_{3}{ }^{-}$is reduced to $\mathrm{NO}_{2}{ }^{-}$by nitrate reductase (NR). Under the low nitrogen condition, the expression level of NR was increased in the GmCBS21 transgenic plants compared with the wild type, but the expression level was similar to the wild type under the normal nitrogen condition. $\mathrm{NO}_{2}{ }^{-}$is further reduced to ammonium $\left(\mathrm{NH}_{4}^{+}\right)$by nitrite reductase (NiR). Under the low nitrogen condition, the expression levels of NiR in the GmCBS21 transgenic plants were similar to the wild type, but were increased 
under the normal nitrogen condition. $\mathrm{NH}_{4}{ }^{+}$is further assimilated into amino acids via the glutamine synthetase (GS)/glutamine-2-oxoglutarate aminotransferase (GOGAT) cycle. The expression level of GS1 and GS2 were lower under low nitrogen but higher under normal nitrogen. Another enzyme involved in $\mathrm{NH}_{4}{ }^{+}$assimilation is glutamate dehydrogenase (GDH). The levels of GDH transcripts were much higher in the L1, L3, and L8 lines than in the wild type under both nutrition regimes. Asparagine synthetase (AS) catalyses the ATP-dependent transfer of the amido group of glutamine to a molecule of aspartate to generate glutamate and asparagine [30]. The expression levels of ASN1 were significantly higher in the L1, L3, and L8 lines than in the wild type under the low nitrogen condition, but the expression levels showed no obvious change under the normal nitrogen condition. Two aminotransferase enzymes, aspartate aminotransferase (AspAT) and alanine aminotransferase (AlaAT), are important in amino acid biosynthesis. The expression levels of AspAT were much higher in the L1, L3, and L8 lines than in the wild type under the normal nitrogen condition, but the expression level was similar to the wild type under the low nitrogen condition. Under the normal nitrogen condition, the expression level of AlaAT in the GmCBS21 transgenic plants was similar to that of the wild type. Under the low nitrogen condition, the expression level of AlaAT was lower in root cells and higher in vegetative leaf cells. PEPc is a key component in photosynthesis and is involved in the production of the keto-acid 2-oxoglutarate [31]. Dof1 is involved in the activation of PEPc [32]. Under the normal nitrogen condition, the expression level of PEPc in the GmCBS21 transgenic plants was similar to that of the wild type. Under the low nitrogen condition, the expression level of PEPc was lower. The expression level of Dof1 was higher in the L1, L3, and L8 lines than in the wild type under both nutrition regimes. PII is an N-sensing and regulatory protein [33]. The expression level of PII in the GmCBS21 transgenic plants was similar to that of the wild type. Pyruvate orthophosphate dikinase (PPDK) is involved in a pathway that generates the transport of the amino acid glutamine [34]. Pyruvate kinase (PK) catalyzes the transfer of a phosphate group from phosphoenolpyruvate (PEP) to ADP, yielding one molecule of pyruvate and one molecule of ATP. In root cells, the expression level of PPDK was significantly reduced in the L1, L3, and L8 lines relative to the wild type under the low nitrogen condition and the normal nitrogen condition. The expression level of PK was significantly increased in the L1, L3, and L8 lines relative to the wild type under the normal nitrogen condition. In vegetative leaf cells, the expression level of PK was significantly reduced in the L1, L3, and L8 lines relative to the wild type under the low nitrogen condition and the normal nitrogen condition. PPDK exhibited the opposite response. Amino acid permease (AAP1) is an integral membrane protein that catalyzes $\mathrm{H}^{+}$-coupled amino acid uptake [35]. The expression level of AAP1 was significantly reduced in the L1, L3, and L8 lines relative to the wild type under the low nitrogen condition, but was similar in the wild-type and the L1, L3, and L8 seedlings under the normal nitrogen condition.

Under the low nitrogen condition, all transgenic plants produced high levels of mRNA for AlaAT, $\mathrm{GDH}$, and AS. AlaAT catalyzes the reversible transfer of an amino group from glutamate to pyruvate to form 2-oxoglutarate and alanine. The regulation of AlaAT in several plant species has been studied in response to low oxygen stress light and nitrogen application, and it is involved in both carbon and nitrogen metabolism in plants [36]. Some studies discovered that overexpression of barley AlaAT in Brassica napus resulted in increased yield and biomass under N-limiting conditions compared with control plants [37]. Overexpression of AlaAT in transgenic rice plants with a tissue-specific promoter (OsAnt1) significantly increased biomass and grain yield [38]. Amino acids are catabolized by both glutamate GDH and transaminases. Ammonium is reassimilated by GS and, through the action of AS, is stored in asparagine (Asn). It has been reported that Arabidopsis plants deficient in GDH have shown decreased growth on reduced levels of $C$ [39]. As previously reported, overexpression of AS with a CaMV35S promoter could enhance NUE in Arabidopsis plants; the transgenic plants had higher tolerance to low nitrogen when grown on plates and in the glasshouse [30]. Based on the above analyses, we speculate that overexpression of GmCBS21 in transgenic plants activated the expression of $A l a A T, G D H$, and $A S$ under low nitrogen conditions, thus affecting the low nitrogen tolerance of plants. 


\section{Materials and Methods}

\subsection{Database Searches for the Identification of CBS Domain-Containing Proteins Members in Soybean}

Sequences of Arabidopsis and Oryza sativa CBS domain containing proteins (CDCPs) were retrieved from the Arabidopsis genome database, AIR 9.0 [40], the Rice Genome Annotation Project [41], and used as queries in BLAST searches against the soybean genome database of the Phytozome database) [10]. Sequences were selected for further analysis if the E value was below 1.0. A keyword search was conducted at the Phytozome (v9.0) database [42] for putative soybean CDCPs by searching ontologies with the term (PF00571) of CBS domain. If more than one transcript existed, the primary transcript was selected as representative. Then, we verified these sequences using the Pfam database [43] and SMART program [44]. Soybean-expressed sequence tag (EST) sequences were searched by blastn program in the Gene Indices at DFCI [45] using the transcript sequences of the identified putative soybean CBS genes as queries.

\subsection{Phylogenetic, Motif Recognition, Gene Structure and Chromosomal Location Analyses}

The multiple alignment analysis was performed with ClustalX 1.83 software [46]. Phylogenetic trees were generated by the neighbor-joining (NJ) method and bootstrap analysis (1000 replicates), and phylogenetic analysis was performed using MEGA5 software [47]. The conserved motifs were analyzed using MEME version 4.9.1 [48]. The exon/intron structures of CBS genes were determined by comparing the coding sequences and corresponding genomic sequences in the Gene Structure Display Server (GSDS) [49]. The chromosomal location of soybean CBS genes was generated using Chromosome Visualization Tool (CViT) at the Legume Information System [50].

\subsection{Plant Materials and Growth Condition}

Soybean seeds were germinated and grown hydroponically in half-strength modified Hoagland solution. The seedlings were grown for 10 days until the first trifoliate leaves fully developed, and then were grown with $10 \%$ of the normal $\mathrm{N}$ concentration. Leaves, stems, and roots were harvested separately after $0,0.5,2,6,9$, and $12 \mathrm{~h}$ and 12 days of this treatment. Glycine max plants were used in this study. Pohuang and 84-70 cultivars were kept in our laboratory. T3 transgenic Arabidopsis and wild-type Arabidopsis plants were germinated and grown on MS media. After seven days, the seedlings were divided into two groups. One group of seedlings was transferred to low nitrogen $\left(0.4 \mathrm{mM} \mathrm{NO}_{3}{ }^{-}\right.$ and $0.2 \mathrm{mM} \mathrm{NH}_{4}{ }^{+}$, other ingredients refer to MS basic culture medium) MS media. For evaluation of soil-grown plants, another group of seedlings was transferred to pots containing vermiculite and Hoagland's nutrient solution: $\left(2 \mathrm{mM} \mathrm{Ca}\left(\mathrm{NO}_{3}\right)_{2} \cdot 4 \mathrm{H}_{2} \mathrm{O}, 2.5 \mathrm{mM} \mathrm{KNO} 3,0.5 \mathrm{mM} \mathrm{NH}_{4} \mathrm{NO}_{3}, 0.5 \mathrm{mM}\right.$ $\mathrm{KH}_{2} \mathrm{PO}_{4}, 1 \mathrm{mM} \mathrm{MgSO} \cdot 7 \mathrm{H}_{2} \mathrm{O}, 0.05 \mathrm{mM}$ Fe-EDTA, $0.005 \mathrm{mM} \mathrm{KI}, 0.1 \mathrm{mM} \mathrm{H}_{3} \mathrm{BO}_{3}, 0.1 \mathrm{mM} \mathrm{MnSO} \cdot \mathrm{H}_{2} \mathrm{O}_{\text {, }}$ $0.03 \mathrm{mM} \mathrm{ZnSO}_{4} \cdot 7 \mathrm{H}_{2} \mathrm{O}, 0.0001 \mathrm{mM} \mathrm{CuSO}_{4} \cdot 5 \mathrm{H}_{2} \mathrm{O}, 0.001 \mathrm{mM} \mathrm{Na}_{2} \mathrm{MO}_{4} \cdot 2 \mathrm{H}_{2} \mathrm{O}, 0.0001 \mathrm{mM} \mathrm{CoCl}_{2} \cdot 6 \mathrm{H}_{2} \mathrm{O}$ ) to analyze the basal phenotype under the sufficient nutrient condition. Before bolting, the seedlings were treated with a nutrient solution without nitrogen or sufficient nitrogen. Samples were collected regularly to determine certain physiological indices.

\subsection{RNA Extraction and Gene Expression Assays}

Total RNA was extracted using TriZol reagent (Invitrogen, Carlsbad, CA, USA) and treated with DNaseI (Invitrogen) to avoid genomic DNA contamination. The first-strand cDNAs were synthesized using Superscript II reverse transcriptase (Invitrogen). The gene expression was analyzed by quantitative RT-PCR (qRT-PCR), which was performed with a SYBRR Premix ExTaq ${ }^{\mathrm{TM}}$ II kit (TaKaRa, Dalian, China) on an IQ ${ }^{\mathrm{TM}} 5$ and MyiQ ${ }^{\mathrm{TM}}$ Real-Time PCR Detection Systems (Bio-Rad, Hercules, CA, USA). Table S6 lists the gene-specific and actin primers. The relative expression level is calculated using $2^{-\Delta \Delta C t}$ method. 


\subsection{Prokaryotic Expression and Cystathionine $\beta$-Synthase Activity Assay}

Based on the sequencing results, the codons of the GmCBS21 gene were optimized and the signal peptides were removed. The pCZN1-GmCBS21 plasmid was transduced into BL21 (DE3) competent cells for expression. The purified proteins were analyzed using SDS-PAGE. The cystathionine $\beta$-synthase activity of the purified protein was determined using the ninhydrin coloration method [51]. The absorbance at $454 \mathrm{~nm}$ was read against water and an enzyme-free blank was subtracted. The amount of cystathionine formed was determined relative to a standard, without enzyme, but containing $1 \mu \mathrm{mol}$ of L-cystathionine.

\subsection{Generation of GmCBS21 Transgenic Plants}

The 1464 bp GmCBS21 CDS was PCR amplified from the soybean genotype Pohuang. The cDNA fragment was ligated into the $\mathrm{PCXSN}$ vector driven by the CaMV $35 \mathrm{~S}$ promoter. The hptII gene was used as a selection marker. All of the procedures of the experimental method followed standard molecular techniques. The plant expression vector pCXSN-GmCBS21 was transformed into Arabidopsis (Columbia) by the floral dip method [52] using the Agrobacterium tumefaciens strain GV3101. Seeds were screened using $100 \mathrm{mg}$. $\mathrm{L}^{-1}$ hygromycin. Hygromycin-resistant seedlings were self-pollinated to produce T2 generation seeds. The seedlings of each generation were tested using PCR and RT-PCR to confirm the transgenic status.

\subsection{Quantification of Nitrogen, Soluble Sugar, Amino Acids}

After 14 days of $\mathrm{N}$-limited treatment, plants were collected and rapidly frozen in liquid nitrogen and then stored at $-80^{\circ} \mathrm{C}$ until analysis. Total nitrogen was determined using the Kjeldahl method [53]. Ammonium nitrogen and non-protein nitrogen were determined using colorimetric assays. Nitrate nitrogen was determined using an ultraviolet spectrophotometer. Soluble sugar contents were determined as described by Geiger [54]. For amino acid analysis, whole plants were ground in liquid nitrogen. The samples were hydrolyzed with HCL of $6 \mathrm{~mol} / \mathrm{L}$ and analyzed using an automatic amino acid analyzer. Asparagine and glutamine were determined using HPLC.

\section{Conclusions}

In conclusion, we cloned a function-unknown gene (GmCBS21) from soybean, which had a CBS domain. Our research shows that GmCBS21 does not belong to cystathionine $\beta$-synthase, and is different from other genes of known functions that contain a CBS domain. The GmCBS21 protein is localized to the plasma membrane. Most importantly, overexpression of GmCBS21 increased the tolerance of Arabidopsis under the low nitrogen condition, and it may play an important role in the nitrogen utilization efficiency of soybean. Although the precise molecular basis of improved tolerance to low nitrogen stress of GmCBS21-overexpressing transgenic Arabidopsis plants has not been completely resolved in this report, further characterization of the GmCBS21 protein at the biochemical and molecular levels will provide insight into the exact nature and function of this protein in tolerance to low nitrogen stress.

Supplementary Materials: Supplementary materials can be found at http:/ / www.mdpi.com/ 1422-0067/17/ 5/620/s1.

Acknowledgments: This work was supported by a grant from the National Genetically Modified Organisms Breeding Major Projects (2009ZX08004-005), the National Natural Science Foundation of China (31401942) and the General Financial Grant from the China Postdoctoral Science Foundation (2014M550899). We also thank anonymous reviewers for their constructive comments. We thank LetPub (www.letpub.com) for its linguistic assistance during the preparation of this manuscript.

Author Contributions: Qingnan Hao and Xinan Zhou designed the experiments. Qingnan Hao and Weijuan Shang performed the experiments; Qingnan Hao, Weijuan Shang, Chanjuan Zhang, and Limiao Chen analyzed the data; Haifeng Chen, Songli Yuan, Xiaojuan Zhang, Shuilian Chen, Xinan Zhou contributed reagents/materials/analysis tools; Qingnan Hao and Xinan Zhou wrote the article. All authors read and approved the final manuscript. 
Conflicts of Interest: The authors declare no conflict of interest.

\section{References}

1. Bao, A.; Liang, Z.; Zhao, Z.; Cai, H. Overexpressing of OsAMT1-3, a high affinity ammonium transporter gene, modifies rice growth and carbon-nitrogen metabolic status. Int. J. Mol. Sci. 2015, 16, 9037-9063. [CrossRef] [PubMed]

2. Good, A.G.; Shrawat, A.K.; Muench, D.G. Can less yield more? Is reducing nutrient input into the environment compatible with maintaining crop production? Trends Plant Sci. 2004, 9, 597-605. [CrossRef] [PubMed]

3. Frink, C.R.; Waggoner, P.E.; Ausubel, J.H. Nitrogen fertilizer: Retrospect and prospect. Proc. Natl. Acad. Sci. USA 1999, 96, 1175-1180. [CrossRef] [PubMed]

4. Hao, Q.N.; Zhou, X.A.; Sha, A.H.; Wang, C.; Zhou, R.; Chen, S.L. Identification of genes associated with nitrogen-use efficiency by genome-wide transcriptional analysis of two soybean genotypes. BMC Genom. 2011, 12, 525. [CrossRef] [PubMed]

5. Bateman, A. The structure of a domain common to archaebacteria and the homocystinuria disease protein. Trends Biochem. Sci. 1997, 22, 12-13. [CrossRef]

6. Sintchak, M.D.; Fleming, M.A.; Futer, O.; Raybuck, S.A.; Chambers, S.P.; Caron, P.R.; Murcko, M.A.; Wilson, K.P. Structure and mechanism of inosine monophosphate dehydrogenase in complex with the immunosuppressant mycophenolic acid. Cell 1996, 85, 921-930. [CrossRef]

7. Schmidt-Rose, T.; Jentsch, T.J. Reconstitution of functional voltage-gated chloride channels from complementary fragments of CLC-1. J. Biol. Chem. 1997, 272, 20515-20521. [CrossRef] [PubMed]

8. Woods, A.; Cheung, P.C.; Smith, F.C.; Davison, M.D.; Scott, J.; Beri, R.K.; Carling, D. Characterization of AMP-activated protein kinase beta and gamma subunits. Assembly of the heterotrimeric complex in vitro. J. Biol. Chem. 1996, 271, 10282-10290. [PubMed]

9. Kemp, B.E. Bateman domains and adenosine derivatives form a binding contract. J. Clin. Investig. 2004, 113, 182-184. [CrossRef] [PubMed]

10. Kushwaha, H.R.; Singh, A.K.; Sopory, S.K.; Singla-Pareek, S.L.; Pareek, A. Genome wide expression analysis of CBS domain containing proteins in Arabidopsis thaliana (L.) Heynh and Oryza sativa L. reveals their developmental and stress regulation. BMC Genom. 2009, 10, 200. [CrossRef] [PubMed]

11. Ignoul, S.; Eggermont, J. CBS domains: Structure, function, and pathology in human proteins. Am. J. Physiol. Cell Physiol. 2005, 289, C1369-C1378. [CrossRef] [PubMed]

12. Ok, S.H.; Yoo, K.S.; Shin, J.S. CBSXs are sensor relay proteins sensing adenosine-containing ligands in Arabidopsis. Plant Signal. Behav. 2012, 7, 664-667. [CrossRef] [PubMed]

13. Zhu, Q.L.; Li, M.Y.; Liu, G.D.; Li, Y.D.; Sui, S.Z.; Guo, T.Y. Molecular characterization and functional prediction of a novel leaf SAG encoding a CBS-domain-containing protein from Coleus blumei. Chin. J. Biochem. Mol. Biol. 2007, 23, 249-255.

14. Singh, A.K.; Kumar, R.; Pareek, A.; Sopory, S.K.; Singla-Pareek, S.L. Overexpression of rice CBS domain containing protein improves salinity, oxidative, and heavy metal tolerance in transgenic tobacco. Mol. Biotechnol. 2012, 52, 205-216. [CrossRef] [PubMed]

15. Wang, X.; Ren, X.; Zhu, L.; He, G. OsBi1, a rice gene, encodes a novel protein with a CBS-like domain and its expression is induced in responses to herbivore feeding. Plant Sci. 2004, 166, 1581-1588. [CrossRef]

16. French, A.P.; Mills, S.; Swarup, R.; Bennett, M.J.; Pridmore, T.P. Colocalization of fluorescent markers in confocal microscope images of plant cells. Nat. Protoc. 2008, 3, 619-628. [CrossRef] [PubMed]

17. Collart, F.R.; Osipiuk, J.; Trent, J.; Olsen, G.J.; Huberman, E. Cloning and characterization of the gene encoding IMP dehydrogenase from Arabidopsis thaliana. Gene 1996, 174, 217-220. [CrossRef]

18. Emanuelle, S.; Hossain, M.I.; Moller, I.E.; Pedersen, H.L.; van de Meene, A.M.; Doblin, M.S.; Koay, A.; Oakhill, J.S.; Scott, J.W.; Willats, W.G.; et al. SnRK1 from Arabidopsis thaliana is an atypical AMPK. Plant J. 2015, 82, 183-192. [CrossRef] [PubMed]

19. Lv, Q.D.; Tang, R.J.; Liu, H.; Gao, X.S.; Zheng, H.Q.; Zhang, H.X. Cloning and molecular analyses of the Arabidopsis thaliana chloride channel gene family. Plant Sci. 2009, 176, 650-661. [CrossRef] 
20. Hechenberger, M.; Schwappach, B.; Fischer, W.N.; Frommer, W.B.; Jentsch, T.J.; Steinmeyer, K. A family of putative chloride channels from Arabidopsis and functional complementation of a yeast strain with a CLC gene disruption. J. Biol. Chem. 1996, 271, 33632-33638. [CrossRef] [PubMed]

21. Wang, S.; Su, S.Z.; Wu, Y.; Li, S.P.; Shan, X.H.; Liu, H.K.; Wang, S.; Yuan, Y.P. Overexpression of maize chloride channel gene ZmCLC-D in Arabidopsis thaliana improved its stress resistance. Biol. Plant. 2015, 59, 55-64. [CrossRef]

22. Cao, Y.; Schubert, K.R. Molecular cloning and characterization of a cDNA encoding soybean nodule IMP dehydrogenase. Biochim. Biophys. Acta 2001, 1520, 242-246. [CrossRef]

23. Li, W.Y.; Wong, F.L.; Tsai, S.N.; Phang, T.H.; Shao, G.; Lam, H.M. Tonoplast-located GmCLC1 and GmNHX1 from soybean enhance $\mathrm{NaCl}$ tolerance in transgenic bright yellow (BY)-2 cells. Plant Cell Environ. 2006, 29, 1122-1137. [CrossRef] [PubMed]

24. Wong, T.H.; Li, M.W.; Yao, X.Q.; Lam, H.M. The GmCLC1 protein from soybean functions as a chloride ion transporter. J. Plant Physiol. 2013, 170, 101-104. [CrossRef] [PubMed]

25. McAllister, C.H.; Beatty, P.H.; Good, A.G. Engineering nitrogen use efficient crop plants: The current status. Plant Biotechnol. J. 2012, 10, 1011-1025. [CrossRef] [PubMed]

26. Ho, C.H.; Lin, S.H.; Hu, H.C.; Tsay, Y.F. CHL1 functions as a nitrate sensor in plants. Cell 2009, 138, 1184-1194. [CrossRef] [PubMed]

27. Zhang, H.; Forde, B.G. An Arabidopsis MADS box gene that controls nutrient-induced changes in root architecture. Science 1998, 279, 407-409. [CrossRef] [PubMed]

28. Kiba, T.; Kudo, T.; Kojima, M.; Sakakibara, H. Hormonal control of nitrogen acquisition: Roles of auxin, abscisic acid, and cytokinin. J. Exp. Bot. 2011, 62, 1399-1409. [CrossRef] [PubMed]

29. Takei, K.; Ueda, N.; Aoki, K.; Kuromori, T.; Hirayama, T.; Shinozaki, K.; Yamaya, T.; Sakakibara, H. AtIPT3 is a key determinant of nitrate-dependent cytokinin biosynthesis in Arabidopsis. Plant Cell Physiol. 2004, 45, 1053-1062. [CrossRef] [PubMed]

30. Lam, H.M.; Wong, P.; Chan, H.K.; Yam, K.M.; Chen, L.; Chow, C.M.; Coruzzi, G.M. Overexpression of the ASN1 gene enhances nitrogen status in seeds of Arabidopsis. Plant Physiol. 2003, 132, 926-935. [CrossRef] [PubMed]

31. Coruzzi, G.M.; Zhou, L. Carbon and nitrogen sensing and signaling in plants: emerging "matrix effects". Curr. Opin. Plant Biol. 2001, 4, 247-253. [CrossRef]

32. Yanagisawa, S. Dof1 and Dof2 transcription factors are associated with expression of multiple genes involved in carbon metabolism in maize. Plant J. 2000, 21, 281-288. [CrossRef] [PubMed]

33. Ferrario-Mery, S.; Besin, E.; Pichon, O.; Meyer, C.; Hodges, M. The regulatory PII protein controls arginine biosynthesis in Arabidopsis. FEBS Lett. 2006, 580, 2015-2020. [CrossRef] [PubMed]

34. Taylor, L.; Nunes-Nesi, A.; Parsley, K.; Leiss, A.; Leach, G.; Coates, S.; Wingler, A.; Fernie, A.R.; Hibberd, J.M. Cytosolic pyruvate, orthophosphate dikinase functions in nitrogen remobilization during leaf senescence and limits individual seed growth and nitrogen content. Plant J. 2010, 62, 641-652. [CrossRef] [PubMed]

35. Rolletschek, H.; Hosein, F.; Miranda, M.; Heim, U.; Gotz, K.P.; Schlereth, A.; Borisjuk, L.; Saalbach, I.; Wobus, U.; Weber, H. Ectopic expression of an amino acid transporter (VfAAP1) in seeds of Vicia narbonensis and pea increases storage proteins. Plant Physiol. 2005, 137, 1236-1249. [CrossRef] [PubMed]

36. Miyashita, Y.; Dolferus, R.; Ismond, K.P.; Good, A.G. Alanine aminotransferase catalyses the breakdown of alanine after hypoxia in Arabidopsis thaliana. Plant J. 2007, 49, 1108-1121. [CrossRef] [PubMed]

37. Hawkesford, M.J.; Barraclough, P. Biotechnological Approaches to Improving Nitrogen Use Efficiency in Plants: Alanine Aminotransferase as a Case Study; John Wiley \& Sons, Inc.: Hoboken, NJ, USA, 2011.

38. Shrawat, A.K.; Carroll, R.T.; DePauw, M.; Taylor, G.J.; Good, A.G. Genetic engineering of improved nitrogen use efficiency in rice by the tissue-specific expression of alanine aminotransferase. Plant Biotechnol. J. 2008, 6, 722-732. [CrossRef] [PubMed]

39. Miyashita, Y.; Good, A.G. NAD(H)-dependent glutamate dehydrogenase is essential for the survival of Arabidopsis thaliana during dark-induced carbon starvation. J. Exp. Bot. 2008, 59, 667-680. [CrossRef] [PubMed]

40. Arabidopsis genome database, AIR 9.0. Avaliable online: http://www.arabidopsis.org/ (accessed on 1 March 2016).

41. Rice Genome Annotation Project. Avaliable online: http://rice.plantbiology.msu.edu/index.shtml (accessed on 1 March 2016). 
42. Phytozome (v9.0) database. Avaliable online: http://www.phytozome.net (accessed on 1 March 2016).

43. The Pfam database. Avaliable online: http://Pfam.sanger.ac.uk/ (accessed on 1 March 2016).

44. Letunic, I.; Doerks, T.; Bork, P. SMART 7: Recent updates to the protein domain annotation resource. Nucleic Acids Res. 2012, 40, D302-D305. [CrossRef] [PubMed]

45. The Gene Indices. Avaliable online: http://compbio.dfci.harvard.edu/tgi/ (accessed on 1 March 2016).

46. Thompson, J.D.; Gibson, T.J.; Plewniak, F.; Jeanmougin, F.; Higgins, D.G. The CLUSTAL_X windows interface: Flexible strategies for multiple sequence alignment aided by quality analysis tools. Nucleic Acids Res. 1997, 25, 4876-4882. [CrossRef] [PubMed]

47. Hall, B.G. Building phylogenetic trees from molecular data with MEGA. Mol. Biol. Evol. 2013, 30, $1229-1235$. [CrossRef] [PubMed]

48. Bailey, T.L.; Williams, N.; Misleh, C.; Li, W.W. MEME: Discovering and analyzing DNA and protein sequence motifs. Nucleic Acids Res. 2006, 34, W369-W373. [CrossRef] [PubMed]

49. Guo, A.Y.; Zhu, Q.H.; Chen, X.; Luo, J.C. GSDS: A gene structure display server. Yi Chuan 2007, $29,1023-1026$. [CrossRef] [PubMed]

50. Cannon, E.K.; Cannon, S.B. Chromosome visualization tool: A whole genome viewer. Int. J. Plant Genom. 2011, 2011, 373875. [CrossRef] [PubMed]

51. Kimura, H.; Nakagawa, H. Studies on cystathionine synthetase characteristics of purified rat liver enzyme. J. Biochem. 1971, 69, 711-723. [PubMed]

52. Davis, A.M.; Hall, A.; Millar, A.J.; Darrah, C.; Davis, S.J. Protocol: Streamlined sub-protocols for floral-DIP transformation and selection of transformants in Arabidopsis thaliana. Plant Methods 2009, 5, 3. [CrossRef] [PubMed]

53. Kimberly, A.E.; Roberts, M.G. A method for the direct determination of organic nitrogen by the kjeldahl process. Public Health Pap. Rep. 1905, 31, 109-122. [CrossRef] [PubMed]

54. Geiger, M.; Walch-Liu, P.; Engels, C.; Harnecker, J.; Schulze, E.-D.; Ludewig, F.; Sonnewald, U.; Scheible, W.-R.; Stitt, M. Enhanced carbon dioxide leads to a modified diurnal rhythm of nitrate reductase activity in older plants, and a large stimulation of nitrate reductase activity. Plant Cell Environ. 1998. [CrossRef]

(C) 2016 by the authors; licensee MDPI, Basel, Switzerland. This article is an open access article distributed under the terms and conditions of the Creative Commons Attribution (CC-BY) license (http://creativecommons.org/licenses/by/4.0/). 